PEB Échanges, Programme pour la construction et l'équipement de l'éducation 2002/03

\title{
Centres municipaux de formation en Hongrie
}

\section{Lajos Jeney}




\section{CENTRES MUNICIPAUX DE FORMATION EN HONGRIE}

L'article qui suit décrit certains des problèmes auxquels sont confrontés ceux qui en Hongrie prennent les décisions concernant les équipements de l'éducation, indique qui devrait participer aux processus de conception et pour quelle raison, et présente la mise en place des unités fonctionnelles des centres municipaux de formation.

\section{Les prises de décision}

En Hongrie, les autorités locales sont chargées depuis 1990 des établissements d'enseignement public, et en particulier de la conception des locaux. Comme en témoigne la piètre qualité des équipements éducatifs construits depuis le changement de régime politique, il reste encore à définir un processus approprié de prise de décision qui permette de concilier au mieux le respect de l'autonomie des administrations locales avec les conseils de professionnels et le soutien du gouvernement central. Pour la majorité des administrations locales, qui manquent d'information et de savoir-faire professionnel, l'indépendance est synonyme d'abandon.

Des réglementations émanant du gouvernement central et applicables à l'ensemble du pays définissent les codes de construction à suivre et les conditions de base que doivent satisfaire le développement régional et l'urbanisme, mais elles ne permettent pas à elles seules de concevoir ou de construire un bâtiment public dont le fonctionnement soit efficace. Ayant reconnu ce fait, plusieurs administrations ont pris les mesures nécessaires pour élaborer des réglementations techniques et économiques applicables aux établissements ou institutions relevant de leur tutelle. Si, compte tenu des exigences locales et sociales réelles, les décisions sont prises dans le respect des concepts et règles professionnels actuels, alors la qualité des équipements éducatifs, municipaux et culturels ne manquera certainement pas de s'améliorer.

\section{Le processus de conception}

En Hongrie, les centres municipaux de formation sont des établissements publics modernes, conçus pour assurer avec efficience et au moindre coût certes, un enseignement, mais aussi des activités éducatives, culturelles et récréatives, à la fois aux jeunes et aux adultes.

L'élaboration d'un projet de plan de construction d'un centre municipal de formation exige la coopération de diverses parties : les représentants du milieu éducatif et social et de la société civile, les futurs utilisateurs de l'équipement et l'architecte. Les projets de plan établis de cette façon reflètent les exigences locales réelles ; par ailleurs, les critères de base de contrôle de la qualité sont omniprésents au stade de la planification et de la concep-

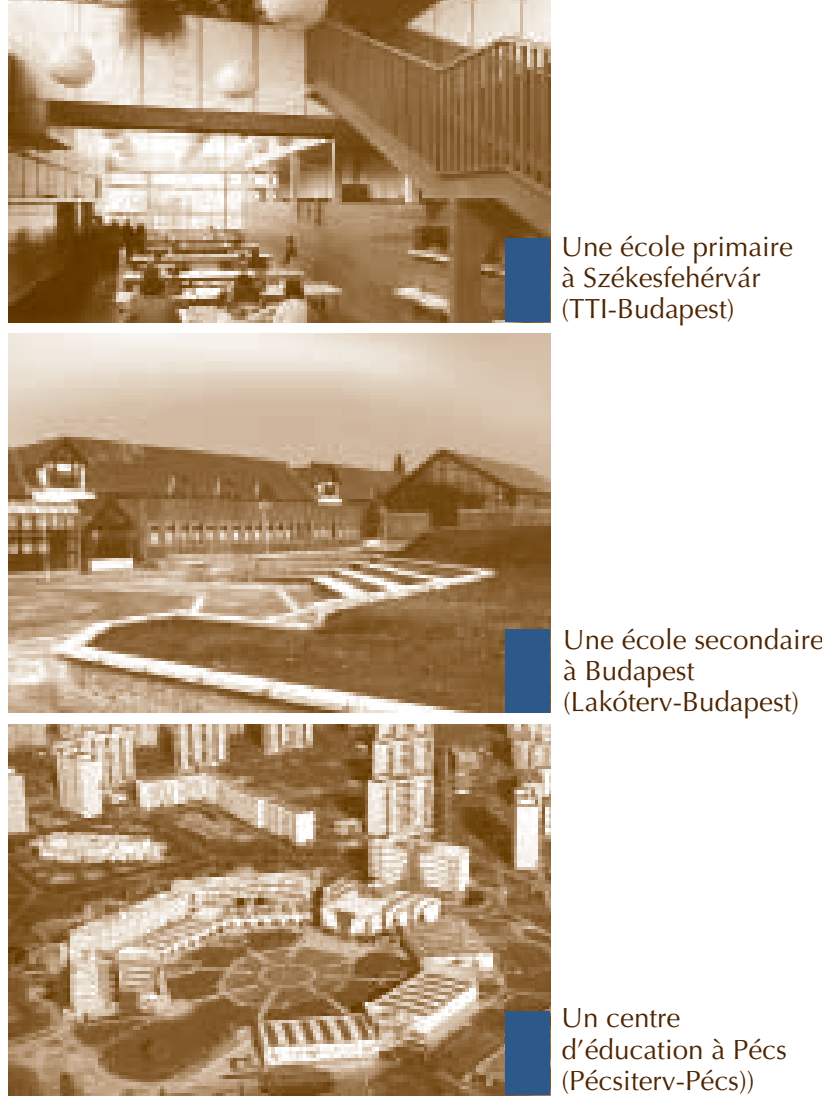

tion. Un bon projet peut produire des bâtiments utiles ayant une valeur architecturale et sociale ; un mauvais projet, en revanche, peut générer des effets nuisibles persistants.

Durant l'étape suivante d'élaboration du plan proprement dit, l'architecte devra continuer de travailler avec les équipes de professionnels qui ont participé à l'élaboration du projet de plan. Du point de vue de la qualité, les usagers doivent avoir la priorité. Dans la mesure où ils sont rarement capables d'exprimer leurs exigences dans le langage spécialisé des architectes, il appartient à ces derniers de formuler ces exigences à la faveur d'un dialogue avec eux ; cet exercice est souvent difficile. Les usagers expriment leurs desiderata en termes de méthodologie, de théorie et de philosophie de l'éducation et les architectes doivent les traduire sous forme de dessins.

Quels usagers doivent participer à ce dialogue ? En théorie, la réponse est très simple : les élèves/étudiants, les enseignants, les parents, les autorités compétentes et les dirigeants de la localité. En réalité, il est rare de pouvoir organiser la participation de tous ces partenaires au dialogue ; en tout état de cause, l'architecte doit rester à l'écoute de l'ensemble des exigences des milieux socioculturels et éducatifs.

\section{Les unités fonctionnelles}

Le centre municipal de formation est un équipement moderne qui est particulièrement adapté aux interrelations entre les outils pédagogiques et l'espace réservé à l'enseignement. Une analyse de l'interaction entre les deux soulève la question de la souplesse et met en lumière plusieurs solutions différentes :

- des espaces à ossature rigide, dotés d'équipements à usage multiple : dans ce cas, le même espace peut avoir diverses utilisations ; 
- des espaces modulables, dotés d'équipements à usage multiple qui permettent d'affecter l'espace à un plus grand nombre d'utilisations que dans le cas précédent;

- des meubles de rangement mobiles pour entreposer les outils pédagogiques. Dans ce cas, il faut accorder une attention particulière à la conception des aires de circulation entre les salles (revêtement approprié des sols, par exemple) ainsi qu'à la conception pratique des portes (des portes sans seuil, par exemple).

L'espace modulable pose non seulement un problème technique, mais également la question de l'utilisation. Ainsi, lorsque les salles séparées par des cloisons mobiles doivent servir de classes, il convient de tenir compte du degré limité d'insonorisation.

L'harmonie entre le mobilier et les installations, l'équipement et la conception architecturale déterminent la qualité de chaque unité fonctionnelle et influent sur son utilité pratique.

Les salles de classe devraient être pourvues des équipements nécessaires pour l'utilisation de matériels audiovisuels commandés à distance (à partir d'un studio central) et disposer de leur propre casier pour entreposer ce matériel ainsi que celui de démonstration. L'aménagement des salles de classe spécialisées dépend pour l'essentiel de la localisation des "points fixes » pour l'arrivée d'eau, de gaz, d'électricité, etc. Les matériels pédagogiques destinés à I'ensemble des disciplines enseignées sont entreposés dans une seule salle située au centre, où les enseignants spécialisés disposent de postes de travail personnels pour faire leurs préparations et leurs travaux de recherche.

La bibliothèque sert non seulement à entreposer les livres mais permet aussi d'accéder à une base de données, à des scanners, à des lecteurs de microfilms, à des supports audiovisuels ainsi qu'à d'autres matériels. La taille et l'agencement du centre $d^{\prime}$ information, de la salle de lecture et d'autres aires de travail dans la bibliothèque ainsi que l'insonorisation et l'éclairage sont des éléments importants pour bien utiliser l'équipement. Le sol, les murs et le plafond sont conçus principalement en fonction de critères acoustiques.

Un atelier à usage général doit être adapté au travail de divers matériaux (bois, métal, matériaux synthétiques, papier, etc.) et doté d'équipements polyvalents récents pouvant être utilisés à la fois par les jeunes et les adultes. II convient de prévoir des cabines séparées de petite taille où les travaux dangereux tels que la soudure ou la coupe puissent être effectués uniquement sous une supervision permanente. La construction et le revêtement des sols, des murs et des plafonds devraient être conçus de façon à résister aux chocs et à l'usure et à permettre un entretien facile. L'acoustique et la protection de l'environnement sont des aspects qui n'ont pas été pris en considération en Hongrie dans l'évaluation des ateliers et il reste donc beaucoup de travaux à faire dans ce domaine.
Lorsqu'il dépasse une taille donnée, le studio doit constituer une unité indépendante dans l'ensemble immobilier. La production de programmes audiovisuels pose de sérieux problèmes du point de vue de l'acoustique et de l'éclairage artificiel. Le revêtement des sols, des murs et des plafonds doit être conçu de façon à correspondre à des critères précis.

Dans la zone réservée aux activités sportives, le mobilier et l'équipement sont conformes à des réglementations techniques rigoureuses. Le sol, qui constitue la principale ressource pour les activités sportives, doit être conçu avec un soin particulier.

Le gymnase, d'une taille suffisante pour accueillir des compétitions (dans des disciplines aussi diverses que le badminton et le handball) doit être équipé de deux rideaux allant d'un mur à l'autre et du sol au plafond afin de pouvoir répartir l'espace en trois gymnases de plus petite taille destinés aux élèves scolarisés.

En ce qui concerne la salle polyvalente, une analyse minutieuse s'impose pour déterminer les équipements et utilisations possibles. Il convient de repérer les deux utilisations les plus extrêmes et d'équiper la salle pour toutes les autres. Dans l'analyse fonctionnelle, le point de départ doit toujours être l'utilisation qui exige la construction la plus solide. Par exemple, lorsque les possibilités d'utilisation vont des activités sportives aux activités culturelles, telles que le théâtre, il faut alors commencer par concevoir et équiper la salle pour répondre aux exigences des activités sportives.

Le principal avantage d'un local polyvalent est que moyennant un minimum de dépenses supplémentaires pour l'équipement, un seul espace peut utilement satisfaire les exigences de nombreux utilisateurs. Dans la mesure où la polyvalence exige de nombreux équipements, les casiers mobiles de rangement sont encore plus importants. S'agissant du mobilier, il convient de privilégier les estrades empilables et les sièges pouvant être installés et déplacés facilement.

En Hongrie, le système de renseignement sur le site est souvent à tort le laissé-pour-compte des ressources des établissements d'enseignement. Des tableaux d'information doivent être installés à chaque entrée. Ils doivent donner une vue d'ensemble de l'établissement tout entier, présentée sous une forme aisément compréhensible, et indiquer l'endroit où se trouve le lecteur. Le système repose sur un code de couleurs, de chiffres et de lettres.

Sans nécessiter d'énormes ressources, les installations de plein air offrent des espaces et des équipements permettant à la fois aux jeunes et aux adultes de mener diverses activités - enseignement, formation, jeux, repos, loisir et activités sportives.

Article de :

Lajos Jeney, Architecte

Budapest, Hongrie 